\title{
The inhibition of proliferation and migration of glioma spheroids exposed to temozolomide is less than additive if combined with irradiation
}

\author{
FABIAN FEHLAUER ${ }^{1,2}$, MARTINA MUENCH $^{1}$, ECKHARD RICHTER $^{1}$ and DIRK RADES ${ }^{1}$ \\ ${ }^{1}$ Department of Radiation Oncology, UKSH, University of Luebeck, Ratzeburger Allee 160, D-23538 Lübeck; \\ ${ }^{2}$ Department of Radiation Oncology, UKE, University of Hamburg, Martinistr. 52, D-20246 Hamburg, Germany
}

Received September 18, 2006; Accepted November 29, 2006

\begin{abstract}
The aim of this study was to investigate the effect of temozolomide (TZM) in combination with X-rays on proliferation and migration in human glioma spheroids. Multicellular spheroids were derived from GaMg and U87 cell lines. Spheroids were treated with various concentrations of TZM (5 $\mu \mathrm{mol}, 0.025 \mathrm{mmol}, 0.05 \mathrm{mmol}$ ) and irradiation (RT). Proliferation and migration assays were performed. For GaMg spheroids, the proliferation inhibition was $30 \%$ (RT), $71 \%$, $79 \%, 85 \%$ (for various TZM concentrations) and 78\%, 83\%, $90 \%$ following RT+TZM. For U87 spheroids, the inhibition of proliferation was $52 \%$ (RT), 62\%, 78\%, 88\% (TZM), and $73 \%, 87 \%, 92 \%$ (RT+TZM). Inhibition of migration for GaMg was 30\% (RT), 37\%, 63\%, 78\% (TZM), and 56\%, $75 \%, 84 \%$ (RT+TZM). For U87, migration inhibition was $29 \%$ (RT), 48\%, 52\%, 67\% (TZM), and 62\%, 67\%, 73\% (RT+TZM). Radiotherapy enhancement ratio (RER) of

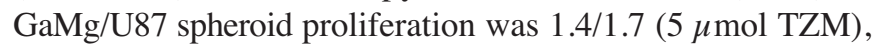
1.3/1.8 (0.025 mmol TZM), and 1.4/1.4 (0.05 mmol TZM). RER for migration of $\mathrm{GaMg} / \mathrm{U} 87$ was 2.2/1.9 (5 $\mu \mathrm{mol} \mathrm{TZM})$, 1.7/1.8 (0.025 mmol TZM), and 1.5/1.4 (0.05 mmol TZM). In terms of inhibition of proliferation and migration, irradiation can lead to an enhancement of the TZM effect in human glioma spheroids, which is less than additive.
\end{abstract}

\section{Introduction}

Gliomas are the most common primary human brain tumours and prognosis is poor, in particular with high-grade tumours such as glioblastoma multiforme (GBM). Intensive surgery followed by radiotherapy with or without adjuvant chemotherapy is considered as standard therapy for primary brain

Correspondence to: Dr Fabian Fehlauer, Department of Radiation Oncology, University Clinic Hamburg-Eppendorf, UKE, University of Hamburg, Martinistr. 52, D-20246 Hamburg, Germany

E-mail: fehlauer@uke.uni-hamburg.de

Key words: glioma, irradiation, temodal, spheroids, proliferation, migration tumours (1). Treatment of brain tumours with adjuvant chemotherapy, mainly 1,3-bis(2-chloroethyl)-1-nitrosourea (BCNU) and 1-(2-chloroethyl)-3-cyclohexyl-1-nitrosourea (CCNU), has only resulted in minor improvements (2-4). Phase I-II clinical studies with temozolomide (TMZ) have demonstrated that this drug crosses the blood-brain barrier and has activity against malignant glioma (5-8).

TMZ in the treatment of primary and recurrent malignant brain tumours is still a topic of clinical trials due to the promising pre-clinical and clinical reports. Recently, Stupp et al found a clinically meaningful increase, by a factor of 2.5 , in the survival rate at two years, from $10 \%$ with radiotherapy alone to $27 \%$ with radiotherapy plus TZM in a randomized phase III trial. The addition of temozolomide to irradiation was associated with improvements in median progression-free survival (6.9 vs. 5.0 month) and overall survival (14.6 vs. 21.1 month) (9).

Since radiotherapy in combination with TMZ might be assumed as gold standard in the treatment of brain malignancies in future (10), it is of interest to investigate further what the pre-clinical effects are on various human glioma cell lines. In a study with the human glioma cell line U373 treated as monolayer cultures, an additive effect was reported for TMZ and X-rays (11). In glioma cells lines U251 and D384, combinations of TMZ and X-rays showed additional as well as supra-additional cytotoxic effects (12).

We have chosen spheroids of U87MG and GaMg glioma cell lines to study the proliverative and migrative behaviour of TZM and irradiation. However, the U87MG cell line contains a functional p53 protein and was described to be sensitive to cytostatic drugs, while the $\mathrm{GaMg}$ cell line was described to be relatively resistant to these compounds $(13,14)$.

Moreover, most of the studies on TZM and its activity against glioma cells were carried out in monolayer cultures $(15,16)$. The spheroid model is a three-dimensional cell culture system that more closely resembles the in vivo situation inside a tumour $(17,18)$. Along the axis of spheroids, steep gradients can exist for cellular oxygen levels, glucose concentration, nutrients, serum-derived growth factors and $\mathrm{pH}$ $(19,20)$. Individual tumour cells growing under these conditions face a different environmental situation depending on their position inside the three-dimensional framework of the spheroid. 
In a three-dimensional cell culture model of the glioma cell lines U87MG and GaMg, a TZM dose-dependent inhibition of tumour growth was found recently, but the role of the clinically relevant combination with irradiation was not investigated. There, the drug was able to induce apoptosis in the core region of glioma U87Mg spheroids, which harbours a functional p53 gene. Thus, TZM might act as an initiator of apoptosis in a glioma spheroid cell culture system (21).

Following these experiments, it was our aim to study the effects of irradiation on TZM-treated glioma cells in terms of tumour cell migration and proliferation in a well-defined three-dimensional spheroid culture system.

\section{Materials and methods}

Cell line spheroids. The human U87 cell line was obtained from Dr J. Ponten, University of Uppsala, Sweden. The GaMg cell line was kindly supplied by Dr R. Bjerkvig, University of Bergen, Norway (22). Both cell lines were grown in Dulbecco's modified Eagle's medium (DMEM) supplemented with 10\% heat-inactivated newborn calf serum, 2\% L-glutamine, 3.2\% nonessential amino acids (alanine, asparagine, aspartic acid, glutamic acid, glycine, proline, serine), penicillin (100 $\mathrm{IU} \mathrm{ml}^{-1}$ ) and streptomycin $\left(100 \mu \mathrm{g} \mathrm{ml}^{-1}\right)$ and kept in standard tissue culture conditions.

Spheroid cultures of both cell lines were formed by liquidoverlay technique (23). In short, exponentially growing monolayers were trypsinized and $5 \times 10^{6}$ cells were seeded in $20 \mathrm{ml}$ of growth medium into $0.8 \%$ agar-coated tissue culture flasks. After 10 days in culture, spheroids with diameter of $\sim 250 \mu \mathrm{m}$ were selected for the experiments. These spheroids do not present central necrosis and have very few hypoxic cells $(24,25)$.

Treatment. Spheroids were irradiated in petri dishes $(60 \mathrm{~mm})$, containing $3 \mathrm{ml}$ of culture medium at a temperature of $37^{\circ} \mathrm{C}$. Due to the known differences in response to irradiation, isoeffective single dose RT was performed using an orthovolt $\mathrm{X}$-ray generator operating at $250 \mathrm{kV}$ and $15 \mathrm{~mA}$, with a $0.5-\mathrm{mm}$ thick $\mathrm{Cu}$ filter and a tube opening of $8 \times 8 \mathrm{~cm}: 10 \mathrm{~Gy}$ for GaMg spheroids and 5 Gy for U87 spheroids. Former experiments of our laboratory explored this RT dosages to be equally effective without sterilising the spheroids (26).

Temozolomide (Temodal ${ }^{\circledR}$, TZM) was obtained from Essex Pharma (Munich, Germany). The drug was added to the culture medium and diluted serially prior to application. TZM was applied in a single dose in concentrations ranging from $5 \mu \mathrm{mol}$ to $0.05 \mathrm{mmol}$ to eight individual spheroids per treatment group. The drug was not removed after application as the half-life of TZM and the first derivative MTIC is $\sim 2 \mathrm{~h}$ (27). Thereafter, equally sized spheroids were removed and exposed to irradiation. Three independent experiments were carried out.

Proliferation assay. Multicellular spheroids were aggregated from single-cell suspension and grown for 7-10 days in liquid overlay culture. Equally sized spheroids with diameters of $\sim 250 \mathrm{~mm}$ were placed in 24-well culture dishes (NUNC, Roskilde, Denmark) base-coated with $0.8 \%$ agar. Each treatment group (sham, irradiation alone, TZM alone, and combinational treatment) consists of 24 glioma spheroids in three independent experiments. To study the spheroidal response to treatment, spheroids were incubated for 18 days. Spheroidal volume growth was monitored daily and spheroid volume was calculated using the equation: $4=3 \pi(D 1+D 2) / 4)^{3}$, where D1 and D2 are the maximal diameters of the spheroids measured in rectangular directions.

In order to measure growth delay, the average time required to reach five times the initial spheroid volume was determined. Specific growth delay (SGD) values were calculated by dividing growth delay of treated samples by the corresponding doubling times of control samples. Treatment-related changes in terms of reduction of the proliferation capacity of spheroids at day 18 were expressed in \% of the control spheroids (100\%). A relative reduction factor was calculated for all treatment groups of both assays (proliferation assay day 18, migration assay day 4) using the equation (control-treatment 1)/treatment 1 and (control-treatment 2)/treatment 2, thereafter (treatment $1 /$ treatment 2). Statistical comparison of the original data was performed using a unpaired sample t-test after analysis of variance. P-values $<0.05$ were considered as significant.

Cellular migration. Each treatment group (sham, irradiation alone, TZM alone, and combinational treatment) consists of 24 glioma spheroids in three independent experiments. After 7 days of stationary culture in agar base-coated dishes, individual multicellular spheroids were placed in 24-well culture dishes. Upon adherence to the solid support, spheroids disassembled and released cells migrated away radially from their initial position. The area $\left(\mathrm{mm}^{2}\right)$ covered by cells was measured every 24 h over a period of 4 days. The area covered by the cells was taken as an indicator of cellular migration ability. Treatmentrelated changes in terms of reduction of the migration area compared to control spheroids were expressed in $\%$.

\section{Results}

Proliferation assay. Proliferation capacity of different TZM concentrations on spheroids of both cell lines was determined. For GaMg spheroids, a significant reduction in spheroid growth was observed following $5 \mu \mathrm{mol}$ TZM of $71 \%$ (specific growth delay, SGD 2.75), $0.025 \mathrm{mmol}$ TZM of 79\% (SGD 4.0), 0.05 mmol TZM of $85 \%$ (SGD 4.5) on day 18. For U87 spheroids, a significant reduction of the spheroid volume was observed following incubation of $5 \mu \mathrm{mol}$ TZM of $62 \%$ (SGD 2.0), $0.025 \mathrm{mmol}$ TZM of $78 \%$ (SGD 3.25), $0.05 \mathrm{mmol}$ TZM of $88 \%$ (SGD 4.25) on day 18.

Following RT alone, GaMg and U87 spheroids showed a significant growth reduction of $30 \%$ (SGD 1.25) and $52 \%$ (SGD 1.75), respectively. Following combined modality treatment, GaMg and U87 spheroids showed an additively significant growth reduction of $78 \%$ (SGD 3.75) and $73 \%$ (SGD 2.7) incubated with $5 \mu \mathrm{mol}$ TZM+RT, 83\% (SGD 5.0) and $87 \%$ (SGD 5.25) incubated with $0.025 \mathrm{mmol}$ TZM+RT, and $90 \%$ (SGD 5.5) and 92\% (SGD 5.5) incubated with 0.05 mmol TZM+RT, respectively (Fig. 1).

Radiotherapy enhancement ratio (RER) of $\mathrm{GaMg} / \mathrm{U} 87$ spheroid proliferation was $1.4 / 1.7(5 \mu \mathrm{mol}$ TZM $), 1.3 / 1.8$ (0.025 mmol TZM), and 1.4/1.4 (0.05 mmol TZM). Table I illustrates the overall outcome of experiments. 

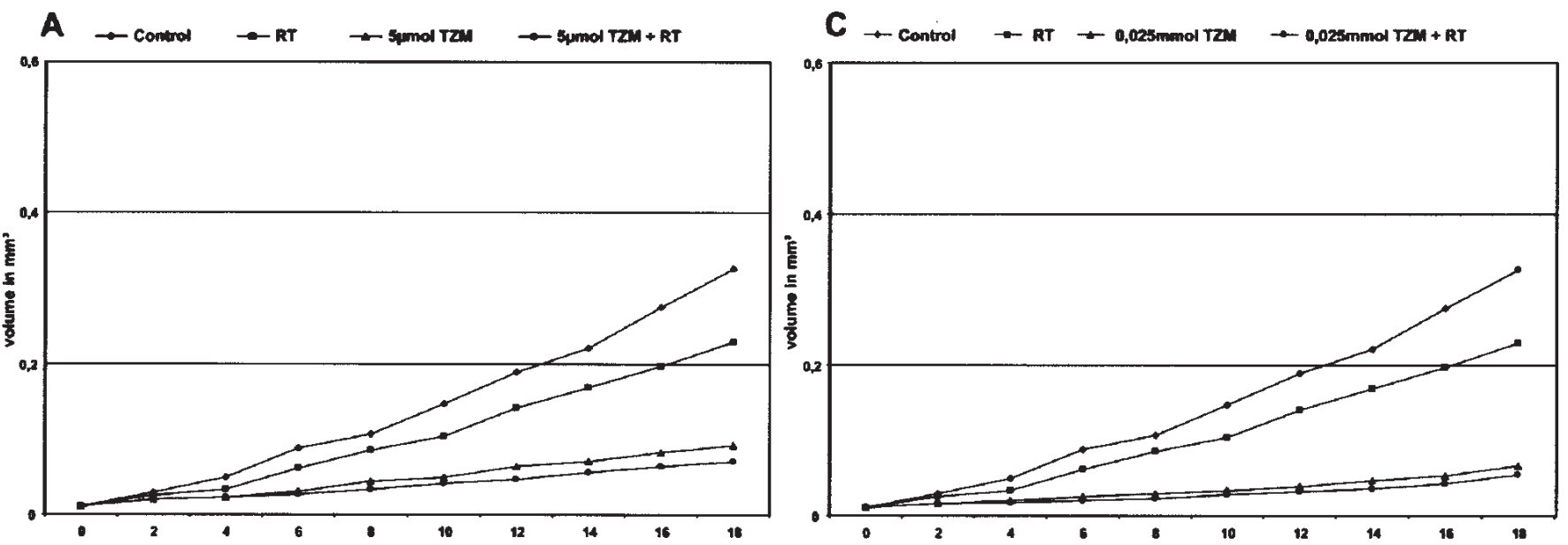

B

D
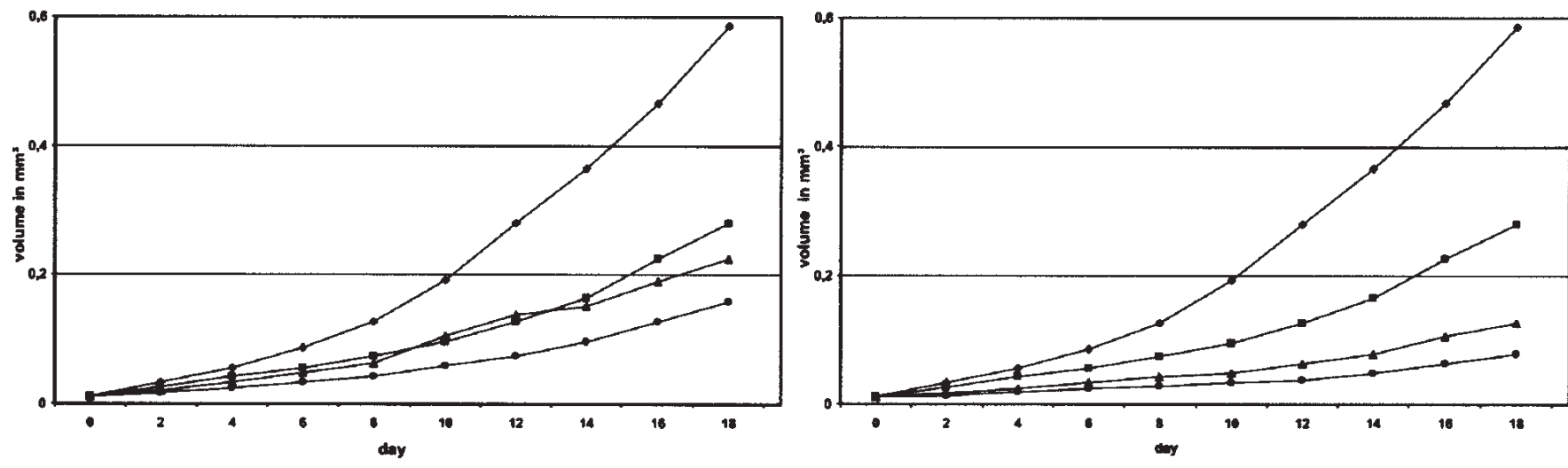

Figure 1. Spheroid proliferation of GaMg and U87 spheroids following single mode and combinational treatment. Each treatment group consists of 24 glioma spheroids in three independent experiments. Illustration of volume growth of GaMg spheroids (A and C) and U87 spheroids (B and D) following irradiation (RT) and temozolomide (TZM): $5 \mu \mathrm{mol}$ TZM (left column), $0.025 \mathrm{mmol}$ TZM (right column). Changes at day 18 were significant $(\mathrm{p}<0.05)$.

Table I. Overall treatment outcome (relative reduction factor at day 18$){ }^{\mathrm{a}}$

\begin{tabular}{|c|c|c|c|c|}
\hline & \multicolumn{2}{|c|}{ Proliferation } & \multicolumn{2}{|c|}{ Migration } \\
\hline & $\mathrm{GaMg}$ & U87 & $\mathrm{GaMg}$ & U87 \\
\hline RT & 0.4 & 1.1 & 0.4 & 0.4 \\
\hline $5 \mu \mathrm{mol} \mathrm{TZM}$ & 2.5 & 1.6 & 0.6 & 0.9 \\
\hline $0.025 \mathrm{mmol} \mathrm{TZM}$ & 3.9 & 3.6 & 1.7 & 1.1 \\
\hline 0.05 mmol TZM & 6.2 & 7.5 & 3.5 & 2.0 \\
\hline $5 \mu \mathrm{mol} \mathrm{TZM}+\mathrm{RT}$ & $3.6(\mathbf{1 . 4 )}$ & 2.7 (1.7) & $1.3(2.2)$ & $1.7(\mathbf{1 . 9})$ \\
\hline $0.025 \mathrm{mmol} \mathrm{TZM}+\mathrm{RT}$ & $4.9(\mathbf{1 . 3})$ & $6.5(\mathbf{1 . 8})$ & $2.9(1.7)$ & $2.0(\mathbf{1 . 8})$ \\
\hline 0.05 mmol TZM+RT & 8.9 (1.4) & 10.9 (1.4) & $5.3(\mathbf{1 . 5})$ & 2.7 (1.4) \\
\hline
\end{tabular}

${ }^{\text {aRT }}$, irradiation; TZM, temozolomide. Bold numbers: radiotherapy enhancement ratio (RER).

Migration assay. The directorial cell migration from the spheroids was determined for treated and untreated spheroids. Following TZM treatment alone for GaMg spheroids, the tumour outgrowth area was reduced by $37 \%(5 \mu \mathrm{mol}$ TZM), $63 \%$ (0.025 mmol TZM), and 78\% (0.05 mmol), respectively. For U87 spheroids, the migration capacity was reduced by
48\% (5 $\mu \mathrm{mol}$ TZM), 52\% (0.025 mmol TZM), and 67\% (0.05 mmol), respectively.

Following RT alone, GaMg and U87 spheroids showed a significant growth reduction of $30 \%$ and $29 \%$ after 4 days. Following combined modality treatment, GaMg and U87 spheroids showed an additively significant growth reduction of $56 \%$ and $62 \%$ incubated with $5 \mu \mathrm{mol} \mathrm{TZM+RT,} 75 \%$ and $67 \%$ (SGD 5.25) incubated with $0.025 \mathrm{mmol}$ TZM+RT, and $84 \%$ and $73 \%$ incubated with $0.05 \mathrm{mmol}$ TZM+RT, respectively (Fig. 2). RER for migration of $\mathrm{GaMg} / \mathrm{U} 87$ was 2.2/1.9 (5 $\mu \mathrm{mol}$ TZM), $1.7 / 1.8(0.025 \mathrm{mmol} \mathrm{TZM})$, and 1.5/1.4 (0.05 mmol TZM).

\section{Discussion}

Local migration and proliferation activity of the remaining tumour cells following operation were inherent features of glioblastoma multiforme. Spheroid growth reflects the proliferation of tumour cells, while the migration assay measures the ability of the cells organised in three-dimensional structure to migrate and proliferate. Since modern radiotherapy is unable to inactivate glioblastoma growth for a prolonged interval, the temporary irradiation benefit has to be intensified by active drugs, such as temozolomide (TZM).

It is assumed that spheroid cultures of glioma cell lines can better predict the in vivo response than monolayer cultures, 

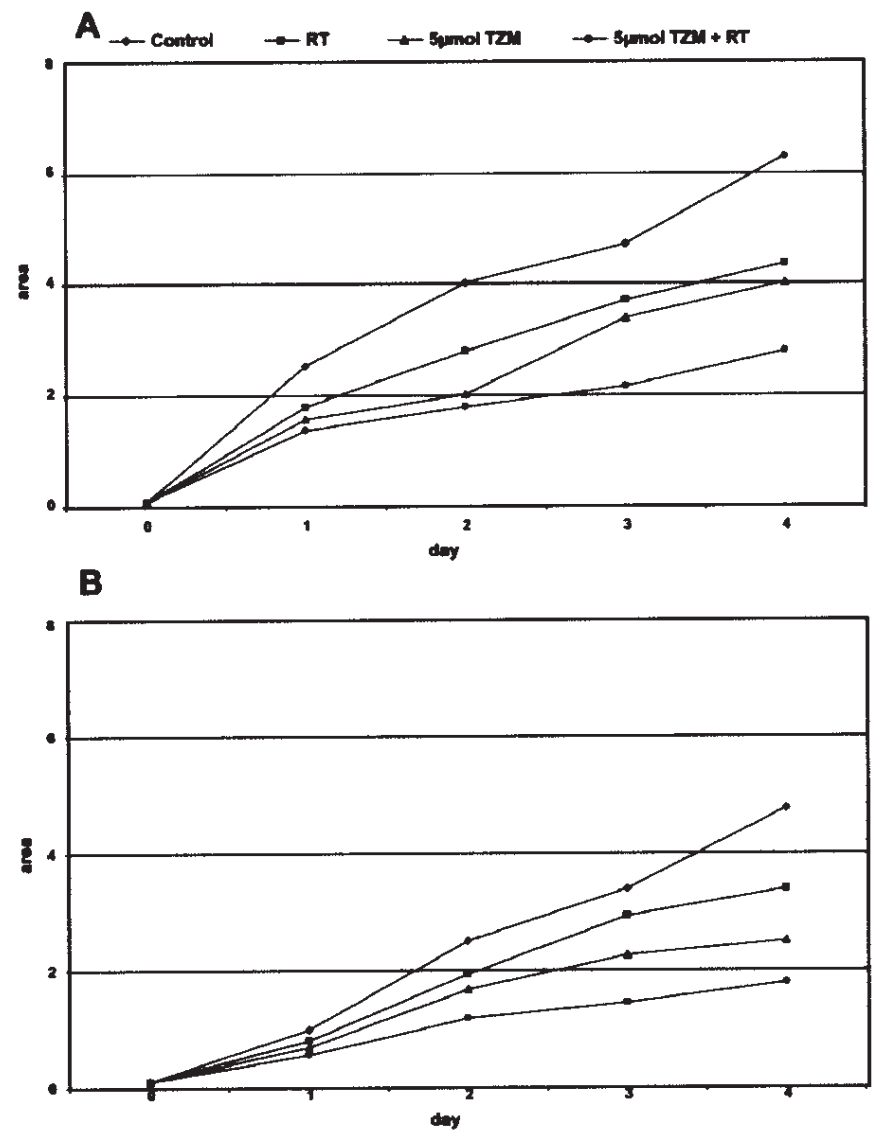

Figure 2. Example for tumor cell migration from GaMg (A) and U87 (B) spheroids following irradiation (RT), temozolomide (TZM), and combinational treatment $(\mathrm{TZM}+\mathrm{RT})$. Area of tumor cell migration $\left(\mathrm{mm}^{2}\right)$. Each treatment group consists of 24 glioma spheroids in three independent experiments. Changes at day 4 were significant $(\mathrm{p}<0.05)$.

since cell-cell contact, variation in cell cycle, altered metabolism, and diffusion of nutrients, oxygen or drugs may influence the outcome $(20,28-31)$. The advantage of cell line spheroids is that they are relatively easy to obtain and to maintain in culture. Treatment-related changes of the growth kinetic of spheroids and the outgrowth of tumour cells present established and reproducible endpoints $(25,32,33)$.

Using spheroids of two biologically different human glioblastoma cell lines, we have shown in this study that irradiation can enhance the cytotoxic effect of TZM, but the exerted effect is less than additive in terms of spheroid growth and migrational behaviour.

Tolerance to TMZ is primarily associated with the activity of the repair protein O6-methylguanine-DNA methyltransferase (MGMT) which removes alkylgroups from DNA (34). Spheroids of GaMg and U87 cells did not show a significant increase of TZM toxicity by co-application of the MGMT inhibitor O6-benzylguanine. Following TZM exposure, apoptosis was induced in spheroids of U87 cells, which express endogenous wild-type p53, but not in spheroids of $\mathrm{GaMg}$ cells (21). This study confirmed former experiments (26), that U87 glioma spheroids were more radiosensitive than GaMg spheroids, but TZM treatment (as well in combination irradiation) was more or less iso-effective in spheroids of both cell lines. Thus, the p53 status of glioma spheroids might influence the response to irradiation more than TZM. Cells with a known functional p53 gene, such as U87Mg spheroids, or an deficient MGMT detoxification system undergo either apoptosis or remain in a senescent state. Both pathways might be able to reduce glioblastoma spheroid growth in terms of cellular proliferation and migration.

In monolayer cultures of U373MG glioma cells, TZM and irradiation provided an additive effect of 2.5- to 3.0-fold increased cell kill (11). Our experiments using spheroid cell cultures led to the finding that irradiation induced an enhancement of various TZM concentrations of 1.3- to 1.8fold growth inhibition for both cell lines. Following combination treatment, a 1.4- to 1.9-fold increased reduction of the tumour cell migration area was noted. However, the effects on spheroid growth and migration of combined treatment of TZM and irradiation are less than additive ( $<2.0$-fold). This less than additive effect on the radiotherapy enhancement ratio might be called increased or enhanced, which reflects the current clinical situation at best (9).

Despite the progress in GBM treatment, the clinical responses are of short duration and barely influence the overall survival. Therefore, pre-studies of irradiation and TZM in combination with other classes of anti-neoplastic drugs are warranted.

\section{Acknowledgements}

Mrs. M. Muench was supported by a grand from the KreitzStiftung, Schleswig-Holstein, Germany.

\section{References}

1. Gonzalez Gonzalez D and Hulshof MCCM: Neurooncology. In: Primary tumors and neurological complications of cancer. Radiotherapy. Twijnstra A, Keyser A and Ongerboer de Visser BW (eds). Elsevier, Amsterdam, pp134-158, 1993.

2. Brock CS and Bowler M: Current perspectives in gliomas. Med Oncol 14: 103-120, 1997.

3. Prados MD and Russo C: Chemotherapy of brain tumors. Semin Surg Oncol 14: 88-95, 1998.

4. De Angelis LM: Benefits of adjuvant chemotherapy in highgrade gliomas. Semin Oncol 30: 15-18, 2003.

5. O'Reilly SM, Newlands ES, Glaser MG, Rice-Edwards JM, Brampton M, Illingworth RD, Richards PG, Kennard C, Colquhoun IR and Lewis P: TZM a new oralcytotoxic chemotherapeutic agent with promising activity against primary brain tumors. Eur J Cancer 29: 940-942, 1997.

6. Bower M, Newlands ES, Bleehen NM, Brada M, Begent RJ, Calvert H, Colquhoun I, Lewis $\mathrm{P}$ and Brampton MH: Multicentre CRC phase II trial of TZM in recurrent or progressive high-grade glioma. Cancer Chemother Pharmacol 40: 484-488, 1997.

7. Stupp R, Dietrich PY, Ostermann Kraljevic S, Kraljevic S, Pica A, Maillard I, Maeder P, Meuli R, Janzer R, Pizzolato G, Miralbell R, Porchet F, Regli L, de Tribolet N, Mirimanoff RO and Leyvraz S: Promising survival for patients with newly diagnosed glioblastoma multiforme treated with concomitant radiation plus TZM followed by adjuvant TZM. J Clin Oncol 20: 1375-1382, 2002.

8. Lanzetta G, Campanella C, Rozzi A, Nappa M, Costa A, Fedele F, Innocenzi G, Gagliardi FM, Salvati M, Minniti G, Frati A, Frati L and Vecchione A: TZM in radio-chemotherapy combined treatment for newly-diagnosed glioblastoma multiforme: phase II clinical trial. Anticancer Res 23: 5159-5164, 2003.

9. Stupp R, Mason WP, van den Bent MJ, Weller M, Fisher B, Taphoorn MJ, Belanger K, Brandes AA, Marosi C, Bogdahn U, Curschmann J, Janzer RC, Ludwin SK, Gorlia T, Allgeier A, Lacombe D, Cairncross JG, Eisenhauer E and Mirimanoff RO: European Organisation for Research and Treatment of Cancer Brain Tumor and Radiotherapy Groups; National Cancer Institute of Canada Clinical Trials Group: Radiotherapy plus concomitant and adjuvant TZM for glioblastoma. N Engl J Med 352: 987-996, 2005 . 
10. De Angelis LM: Chemotherapy for brain tumors - a new beginning. N Engl J Med 352: 1036-1038, 2005.

11. Wedge SR, Porteous JK, Glaser MG, Marcus K and Newlands ES: In vitro evaluation of TZM combined with $\mathrm{X}$-irradiation. Anticancer Drugs 8: 92-97, 1997.

12. van Rijn J, Heimans JJ, van der Berg J, van der Valk P and Slotman BJ: Survival of human glioma cells treated with various combinations of TZM and $\mathrm{x}$-rays. Int $\mathrm{J}$ Radiat Oncol Biol Phys 47: 779-784, 2000.

13. Li H, Lochmuller H, Yong VW, Karpati G and Nalbantoglu J: Adenovirus-mediated wild-type p53 gene transfer and overexpression induces apoptosis of human glioma cells independent of endogenous p53 status. J Neuropathol Exp Neurol 56: 872-878, 1997.

14. Terzis AJ, Thorsen F, Heese O, Visted T, Bjerkvig R, Dahl O, Arnold $\mathrm{H}$ and Gundersen P: Proliferation, migration and invasion of human glioma cells exposed to paclitaxel (Taxol) in vitro. Br J Cancer 75: 1744-1752, 1997.

15. Sankar A, Thomas DG and Darling JL: Sensitivity of short-term cultures derived from human malignant glioma to the anticancer drug TZM. Anticancer Drugs 10: 179-185, 1999.

16. Hirose Y, Berger MS and Pieper RO: p53 effects both the duration of G2/M arrest and the fate of TZM-treated human glioblastoma cells. Cancer Res 61: 1957-1963, 2001.

17. Bjerkvig R, Tonnesen A, Laerum OD and Backlund EO: Multicellular tumor spheroids from human gliomas maintained in organ culture. J Neurosurg 72: 463-475, 1990.

18. Fehlauer F, Stalpers LJ, Panayiotides J, Gonzalez Gonzalez D, Kaaijk P, Leenstra S, van der Valk P and Sminia P: Effect of single dose irradiation on human glioblastoma spheroids in vitro. Oncol Rep 11: 477-485, 2004.

19. Mueller-Klieser W: Multicellular spheroids. A review on cellular aggregates in cancer research. J Cancer Res Clin Oncol 113: $101-122,1987$.

20. Sminia P, Acker H, Eikesdal HP, Kaaijk P, Kaaijk P, Enger P, Slotman B and Bjerkvig R: Oxygenation and response to irradiation of organotypic multicellular spheroids of human glioma. Anticancer Res 23: 1461-1466, 2003.

21. Gunther W, Pawlak E, Damasceno R, Arnold H and Terzis AJ: TZM induces apoptosis and senescence in glioma cells cultured as multicellular spheroids. Br J Cancer 88: 463-469, 2003.

22. Akslen LA, Andersen KJ and Bjerkvig R: Characteristics of human and rat glioma cells grown in a defined medium. Anticancer Res 8: 797-803, 1988.

23. Carlsson J, Nilsson K, Westermark B, Ponten J, Sundstrom C, Larsson E, Bergh J, Pahlman S, Busch C and Collins VP: Formation and growth of multicellular spheroids of human origin. Int J Cancer 31: 523-533, 1983.
24. Mueller-Klieser WF, Freyer JP and Sutherland RM: Influence of glucose and oxygen supply conditions on the oxygenation of multicellular spheroids. Br J Cancer 53: 345-253, 1986.

25. Sutherland R, Freyer J, Mueller-Klieser W, Wilson R, Heacock C, Sciandra J and Sordat B: Cellular growth and metabolic adaptations to nutrient stress environments in tumor microregions. Int J Radiat Oncol Biol Phys 12: 611-615, 1986.

26. Gliemroth J, Feyerabend T, Gerlach C, Arnold $\mathrm{H}$ and Terzis AJ: Proliferation, migration, and invasion of human glioma cells exposed to fractionated radiotherapy in vitro. Neurosurg Rev 26: 198-205, 2003.

27. Kim HK, Lin CC, Parker D, Lim J, Likhari P, Statkevich P, Marco A and Nomeir AA: High-performance liquid chromatographic determination and stability of 5-(3-methyltriazen-1-yl)imidazo-4-carboximide, the biologically active product of the antitumor agent TZM, in human plasma. J Chromatogr B Biomed Sci Appl 703: 225-233, 1997.

28. Sutherland RM: Cell and environment interactions in tumor microregions: the multicell spheroid model. Science 240: 177-184, 1988.

29. Kunz-Schughart LA: Multicellular tumor spheroids: intermediates between monolayer culture and in vivo tumor. Cell Biol Int 23: 157-161, 1999.

30. Santini MT, Rainaldi G and Indovina PL: Multicellular humour spheroids in radiation biology. Int J Radiat Biol 75: 787-799, 1999.

31. Walenta S, Doetsch J, Mueller-Klieser W and Kunz-Schughart LA: Metabolic imaging in multicellular spheroids of oncogenetransfected fibroblasts. J Histochem Cytochem 48: 509-522, 2000.

32. Mahesparan R, Tysnes BB, Read TA, Enger PO, Bjerkvig R and Lund-Johansen M: Extracellular matrix-induced cell migration from glioblastoma biopsy specimens in vitro. Acta Neuropathol 97: 231-239, 1999.

33. Gliemroth J, Zulewski H, Arnold H and Terzis AJ: Migration, proliferation, and invasion of human glioma cells following treatment with simvastatin. Neurosurg Rev 26: 117-124, 2003.

34. Bobola MS, Silber JR, Ellenbogen RG, Geyer JR, Blank A and Goff RD: O6-methylguanine-DNA methyltransferase, O6benzylguanine, and resistance to clinical alkylators in pediatric primary brain tumor cell lines. Clin Cancer Res 11: 2747-2755, 2005. 